\title{
How do drugs for psychiatric disorders work?
}

Received 5 October 2017; Accepted 5 October 2017; First published online 9 November 2017

Key words: antidepressants, antipsychotics, emotion, side-effects, mechanisms.

Commentary on: Moncrieff J (2017). Research on a 'drug-centred' approach to psychiatric drug treatment: assessing the impact of mental and behavioural alterations produced by psychiatric drugs. Epidemiology and Psychiatric Sciences. doi: 10.1017/S2045796017000555.

This challenging question is explored in an article by Professor Joanna Moncrieff in the current edition of this journal. Moncrieff argues that the traditional 'disease-based' concept of drug action, which suggests that drugs act to reverse core underlying pathophysiology, is fundamentally mistaken. She suggests that psychotropic drug action can be better understood using a 'drug-based' approach; this posits that psychotropic drugs work by inducing abnormal mental states which then help individuals deal with the distressing experiences that characterise psychiatric disorders. In assessing Moncrieff's proposal, it is important to remember that key pharmacological treatments in psychiatry were discovered by chance and were often used to generate disease hypotheses at a time when neurobiological knowledge of pathophysiology was essentially nil. For example, the ability of antipsychotic drugs to block dopamine receptors gave rise to the dopamine theory of schizophrenia while the facilitation by tricyclic antidepressants of noradrenaline and serotonin (5-HT) activity led to the monoamine hypothesis of depression (Millan et al. 2015). This was of course simplistic but at the time there were few other clues.

In fact, though dismissed by Moncrieff, modern imaging studies indicate that acute psychosis is indeed associated with increased release of dopamine and this is very likely to explain the antipsychotic actions of dopamine receptor blockers (Howes et al. 2017). However, this is by no means to claim that this effect represents a specific 'reversal' of disease pathology. The origin of the increase in dopamine release is uncertain and anyway the abnormality is in presynaptic release of dopamine rather than at the post-synaptic

* Address for correspondence: C. J. Harmer, University Department of Psychiatry and Oxford Health NHS Trust, Warneford Hospital, Oxford OX3 7JX, UK.

(Email: Catherine.harmer@psych.ox.ac.uk) dopamine receptor where current antipsychotic drugs act. In addition, other important clinical features of schizophrenia such as negative symptoms and cognitive impairment are not linked to dopamine overactivity and of course antipsychotic drugs are not effective for these aspects of the clinical syndrome (Miyamoto et al. 2012). However, it does seem clear that part of the pathophysiology of acute psychosis includes dopamine hyperactivity and this is counteracted pharmacologically by antipsychotic drug treatment.

In contrast, the evidence that clinical depression is associated with impaired serotonin or noradrenaline activity is indeed weak and inconsistent (Cowen \& Browning, 2015). Nevertheless, it is established that patients with depression have negative biases in emotional processing, which are believed to play a key role in the vulnerability to and maintenance of clinical symptomatology (Roiser \& Sahakian, 2013). Intriguingly, these negative biases are rapidly reversed by antidepressant drugs that potentiate 5-HT and noradrenaline function (Harmer et al. 2017). Moreover, this reversal correlates with eventual clinical outcome, suggesting an important therapeutic role for this particular pharmacological action (Tranter et al. 2009). This is not to suggest that 'low 5-HT' causes depression but it does indicate that increasing 5-HT function reverses a pathophysiological process central to the experience of depression. This seems close to the use of $\beta$-adrenoceptor agonists in asthma, described by Moncrieff. Beta-adrenoceptor agonists reverse airway narrowing and help relieve asthma symptoms, but the cause of airway narrowing does not rest with some deficiency in $\beta$-adrenoceptors.

Overall, therefore, the reality of the actions of psychotropic drugs in clinical disorders is more granular and complex than a simple dichotomy into 'drugcentred' and disease-centred' approaches will allow and there are many similarities in this respect between drugs used to treat general medical conditions and those used in psychiatry. As Moncrieff puts it, both 'act on the physiological processes that produce symptoms'. This also explains the relative specificity of psychotropic drugs for different psychiatric conditions. For example, by Moncrieff's account, the sedating and relaxing effects of tricyclic 
antidepressants should make them useful for the treatment of psychosis - however, they are not - presumably because despite their pharmacological similarity to phenothiazines, they lack dopamine receptorblocking properties.

One can only support Moncrieff's call for more knowledge about the subjective effects of psychotropic drug treatment, particularly in the longer-term. However, we would place less emphasis on studies involving subjective reports in healthy volunteers for this purpose. It is not clear whether or not Moncrieff believes that psychiatric conditions are characterised by underlying disturbances or changes in brain states. However, what would seem to be crucial is the interaction between a psychotropic drug and the brain state associated with a particular psychiatric disorder. We then have to examine both the changes in the mental state caused by the drug as well as those caused by changes in the experience of the disorder. This is clearly relevant to the important issue of moral agency also raised by Moncrieff and which has been studied by Ilina Singh in young people receiving stimulant treatment for ADHD. Generally, the children concerned expressed the view that stimulant treatment improved their capacity for the moral agency (Singh, 2012). Whether such an effect would be reported by unaffected individuals using stimulants recreationally seems doubtful.

What can be taken usefully from Moncrieff's 'drugbased' approach? Clinically there is certainly much to recommend it, in that it encourages a holistic interaction with the experience of the patient. From this one can gain a deeper understanding of what it is 'like' to be on a particular medication and the benefits and problems associated with treatment. This can prompt a mutual search for better approaches based on what the patient finds most helpful. The drugbased approach might also lend it itself to exploring the potential utility in psychiatry of psychotropic drugs that produce profound changes in conscious experience, for example, the effect of psilocybin in refractory depression. What the drug-based approach will not do, however, is help us discover more effective and better-tolerated treatments because these will be based mostly on an improved knowledge of pathophysiology. This underscores the need to better define biology, not of whole disorders which were not originally devised on this basis, but of key processes which are found across disorders, such as deficits in reward processing or increased avoidance. Such an approach may allow a more sensible mapping of pathophysiology to experiences and symptoms (Insel, 2014). Ultimately this could lead to a greater precision of prescribing and a wider range of useful treatments. Advances in neuroscience make this a feasible objective; however, for the drug-based approach, it would be the road not taken.

C. J. Harmer* and P. J. Cowen

University Department of Psychiatry and Oxford Health NHS Trust, Warneford Hospital, Oxford OX3 7JX, UK

\section{References}

Cowen PJ, Browning M (2015). What has serotonin to do with depression? World Psychiatry 14, 158-160.

Harmer CJ, Duman RS, Cowen PJ (2017). How do antidepressants work? New perspectives for refining future treatment approaches. Lancet Psychiatry 4, 409-418.

Howes OD, McCutcheon R, Owen MJ, Murray RM (2017). The role of genes, stress, and dopamine in the development of Schizophrenia. Biological Psychiatry 81, 9-20.

Insel TR (2014). The NIMH Research Domain Criteria (RDoC) Project: precision medicine for psychiatry. American Journal of Psychiatry 171, 395-397.

Millan MJ, Goodwin GM, Meyer-Lindenberg A, Ove Ögren S (2015). Learning from the past and looking to the future: emerging perspectives for improving the treatment of psychiatric disorders. European Neuropsychopharmacology 25, 599-656.

Miyamoto S, Miyake N, Jarskog LF, Fleischhacker WW, Lieberman JA (2012). Pharmacological treatment of schizophrenia: a critical review of the pharmacology and clinical effects of current and future therapeutic agents. Molecular Psychiatry 17, 1206-1227.

Roiser JP, Sahakian BJ (2013). Hot and cold cognition in depression. CNS Spectrums 18, 139-149.

Singh I (2012). Not robots: children's perspectives on authenticity, moral agency and stimulant drug treatments. Journal of Medical Ethics 39, 359-366.

Tranter R, Bell D, Gutting P, Harmer C, Healy D, Anderson IM (2009) The effect of serotonergic and noradrenergic antidepressants on face emotion processing in depressed patients. Journal of Affective Disorders 118, 87-93. 